\title{
Birirgit Mìiller
}

\author{
Der MYthos vom Paten Ossi \\ Debisch-deutsche Vorurteile bnol die Erfahrungen mit der \\ Marktwirtschaft in drei Ostberliner Petrieben
}

»Nach der Wende hätte, wie man so schön sagt, eine Bestandsaufnahme gemacht werden müssen: Welches Niveau hat unsere Industrie, oder beziehungsweise die Industrie bei uns - .. denn nen gewissen Stand hatten wir ja auch, sonst hätten wir ja nicht so lange existieren können. Diesen Stand hätte man müssen - sagen wir mal - festhalten und darauf aufbauen, modernisieren, also zu höherer Produktivität führen. Aber all das ist nicht gemacht worden und mit einer Nonchalance. Ich möchte sogar behaupten - ich behaupte jetzt - das habe ich vor Jahresfrist und vor anderthalb Jahren auch schon behauptet, was jetzt auch andere behaupten, daß ganz bewußt nicht dieser Status Quo gehalten worden ist, daß nicht darauf aufgebaut worden ist, um sich ganz bewußt Konkurrenz, eventuell vorhandene und eventuell entstehende Konkurrenz, vom Halse zu schaffen und alles erst mal auf einen Nullpunkt zu fahren, damit man das für Null ouvert einsacken kann. Und dann ohne groß zu investieren einen Profit machen.... Das tritt immer deutlicher zu Tage. Es sagen jetzt auch schon ennsthafte Leute, wie der Her de Maiziere es gestern hat durchblicken lassen: das ist alles diktiert worden. Man wollte es gar nicht, daß man hier, sagen wir mal, hätte in echt briiderlicher Gemeinschaft das festgehalten, was schon vorhanden war und darauf aufgebaut. Das wäre den West- also dem Altbundesbürger und überhaupt dem ganzen Deutschland billiger gekommen, als das, was es jetzt anfängt zu kosten. Dem Unternehmer bringt es fette Profite, aber der ganzen Sache hätten wir viele, viele hundert Millionen, wenn nicht Milliarden Kosten ersparen können.«(Facharbeiter, Stanex, 18.4. 1991)

Enttäuschung, das Gefühl betrogen worden zu sein und an die Westdeutschen die politische Kontrolle über die ostdeutsche Wirtschaft verloren zu haben, sprechen aus den Worten dieses Facharbeiters aus einem der Betriebe der ehernaligen DDR, die heute ernsthafte ökonomische Probleme haben. Seine Aussage mag sich drastisch anhören, aber sie ist nicht ungewöhnlich für Meinungen, die man in den Büros und Werkstätten von ostdeutschen Betrieben hören kann. Ich möchte diese Aussagen zum Ausgangspunkt nehmen, um zu untersuchen, inwiefern die Vorurteile, die West- und Ostdeutsche wechselseitig entwickelten, mit ihrer Einstellung zur Arbeit und den unterschiedlichen Erfahrungen des Arbeitslebens in den letzten vierzig Jahren zu tun haben. Auf der Basis von Marerial, das ich in drei Betrieben in Ostberlin sammeln konnte, versuchte ich nachzuvollziehen, wo die Gründe für den Optimismus lagen, mit dem die Ostdeutschen der Marktwirtschaft beigetreten sind. Wie haben Vorstellungen über eine gerechte Wirtschaft die ersten wirtschaftlichen Weichenstellungen nach dem Fall der Mauer beeinflußt? Wie könnte die Konfrontation von zwei 
gegensätzlichen Arbeitskulturen von einem Problem zu einen kreativen Prozeß werden?

Nach dem Fall der Mauer hatten viele Ostdeutsche große Hoffnungen in die westdeutsche soziale Marktwirtschaft gesetzt. Sie war von vielen während der vierzig Jahre der Trennung als ein Wirtschaftssystem idealisiert worden. Auf der anderen Seite der Mauer hatten die Westdeutschen die Unterschiede zu der ostdeutschen Gesellschaft ausschließlich mit westdeutschen Kriterien gemessen und beurteilt und waren davon ausgegangen, daß sich die ostdeutsche Wirtschaft dank der selbstheilenden Kräfte des Marktes schon der westdeutschen anpassen würde.

Der Kollaps der ostdeutschen Wirtschaft, vor allem seit dem 1. Juli 1990, der Bankrott von tausenden von Betrieben und die Massenarbeitslosigkeit überraschten die meisten Ostdeutschen, untergruben ihr Selbstbild und die Vorstellungen, die sie sich von ihrer Umwelt gemacht hatten. Mit ihren Arbeitsplätzen verloren sie auch komplexe gesellschaftliche Rollen, die ihnen von der offiziellen realsozialistischen Politik zugeteilt worden waren. Die, die in den Betrieben bleiben konnten, mußten an ihrem Arbeitsplatz - mehr als in jedem anderen Bereich ihres Lebens - ihren gewohnten Lebensrhythmus, ihre Autoritätsbeziehungen und ihr Verhältnis zu Kollegen und Geschäftspartnern in Frage stellen.

Gleichzeitig wurden hohe Anforderungen an ihre Leistungsbereitschaft gestellt, ohne daß ihnen dafür im Gegenzug ihr Arbeitsplatz sicher gewesen wäre und sie mehr verdient hätten. Sie begannen daher, sich zunehmend im Gegensatz zu dem Bild zu definieren, das sie sich von den Westdeutschen im allgemeinen machten. Anschuldigungen gegen die Westdeutschen dienten manchmal auch als Rechtfertigung für die eigenen Probleme. Der ostwestdeutsche Dialog wurde immer emotionaler und vorurteilsgeladener. Am Ende des Jahres 1990 charakterisierten viele Ostdeutsche, mit denen ich innerhalb und außerhalb der Fabriken sprach, die Westdeutschen als sozial isoliert, besessen von ihrer Arbeit, unfähig zu teilen und als gleichgültig gegenüber den Entwicklungen in der ehemaligen DDR.

Das Bild vom Westen wurde Teil von dem, was Sabel (1982) die »Weltsicht« der Ostdeutschen nennen würde. Weltsichten, wie er sie definiert, sind sowohl normativ als auch explikativ. Sie sind nicht nur Modelle der Welt, sondern auch fiur die Welt (Sabel 1982,14). Die Beziehung zwischen Weltsicht und sozialer Realität ist analog zu der Beziehung zwischen Theorie und Praxis. Weltsichten und Theorien müssen beide ihre Nützlichkeit in der Praxis beweisen. So enthält jede Weltsicht, wie jede interessante Theorie, einen Anteil von objektiver Wahrheit über die soziale Arbeitsteilung oder die Sozialstruktur, auf die sie sich bezieht. Aber dies bedeutet weder, daß eine Weltsicht die ganze Wahrheit über die Welt darstellen würde, 
noch daß nur eine Weltsicht einer gegebenen Situation angemessen wäre (ebd.,12).

Das Bild vom Westen hatte die alte politische Weltkarte des »Schwarzen Kanals« des DDR-Fernsehens als Hintergrund. Das neu/alte negative Bild vom Westen war nicht realistischer als das vorangegangene extrem positive Bild. Eine Ideologie kam wieder zum Vorschein, an die niemand, noch nicht einmal die herbsten Kritiker des kapitalistischen Modells bruchlos geglaubt hatten. Sie diente weniger dazu, die westdeutsche Realität zu erklären und zu verstehen, sondern sie bezog sich auf die ostdeutsche Realität. Sie entsprach der besonderen Erfahrung einer Gesellschaft, die von der Illusion erwachte, ihr Schicksal in die eigenen Hände zu nehmen, und die sich stattdessen an die Rahmenbedingungen einer bestehenden anderen Sozialordnung anzupassen hatte.

Die raschen Veränderungen im Bild des Westens und die politischen und wirtschaftlichen Entwicklungen hatten entscheidende Auswirkungen auf meine Feldforschung in Ostberliner Betrieben. Im Frühling und Sommer 1990 schien mein Interesse als westliche Anthropologin dem Bedürfnis der Beschäftigten entgegenzukommen, sich selbst zu erklären und ihr Selbstbild in den Kommentaren und Reaktionen eines Westlers gespiegelt zu sehen. Nach der Vereinigung im Herbst 1990 war ich nützlich als Mittlerin und Übersetzerin der Regeln und Gesetze der Bundesrepublik Deutschland. Sie nahmen mich als Repräsentantin und Sprecherin für die westdeutsche Kultur an, die sie noch kaum aus eigener Anschauung kannten, betonten ihre Eigenheiten und setzten sich von dem ab, was sie als westdeutsches Vorurteil betrachteten. Im Frühling 1991 wurde ich schließlich der Wessi, der nicht ganz so schlimm ist wie die anderen, und noch im Frühjahr 1993, während ich diese Zeilen schreibe, muß ich immer wieder neu zu den halb ernsthaften, halb scherzhaften Sprüchen Stellung nehmen, daß ich für die Werksleitung spioniere.

Als ich meine Feldforschungen im Mai 1990 begann, war ich erstaunt über die Offenheit und Neugierde meiner Gesprächspartner im Ministerium für Leichtmaschinenbau der DDR, die die Kontakte zu den Direktoren von zwei ehemaligen volkseigenen Betrieben ermöglichten. Ein dritter Betrieb kam im Sommer 1991 hinzu, als die beiden ersten mangels westlichen Käuferinteresses bankrott zu gehen drohten. Um die dort arbeitenden Menschen kennenzulernen, suchte ich in den drei Betrieben jeweils eine Abteilung mit nicht mehr als 100 Mitarbeitern in Verwaltung und Produktion aus. Diese drei Abteilungen stellten Güter her, die in der DDR-Wirtschaft höchst begehrt gewesen waren und die es in ihrer Qualität durchaus mit westlichen Gütern aufnehmen konnten. 
In dem ersten Betrieb, Stanex, der im Juli 1990 noch 2000 Beschäftigte hatte, untersuchte ich einen Betriebsteil mit 100 Mitarbeitern und einer eigenen Verwaltungsstruktur, der Fertigungsautomaten herstellt und schon zu Zeiten der Planwirtschaft fast wie ein eigenständiger Betrieb innerhalb des VEB funktionierte. Er war in den sechziger Jahren aus einem Forschungsinstitut hervorgegangen, das sich mit der Entwicklung von Rationalisierungtechniken - das sind Vorrichtungen und Maschinen zur Rationalisierung der Fertigung - beschäftigte. Im Juni 1991 wurde der Gesamtbetrieb Stanex von einer Immobilienfirma unter der Auflage gekauft, $250 \mathrm{Be}$ schäftigte von den 2000 weiterzubeschäftigen. Der Käufer ermunterte seinerseits die Direktoren der Betriebsteile, sich mit ihren Bereichen auszugründen und die Beschäftigten zu übernehmen. Der von mir untersuchte Bereich wurde eine eigene GmbH, in der noch 62 Leute arbeiten. Diese gehört jetzt dem ehemaligen Direktor, der von der Immobilienfirma Produktionsflächen und Maschinen mietet.

Der zweite Betrieb, Taghell, entstand aus einem Familienbetrieb, der, bis er 1972 in einen VEB umgewandelt wurde, alle Phasen der schleichenden Verstaatlichung durchlaufen hatte. Bis 1989 waren die dort gefertigten Messingleuchten, obwohl sie sehr teuer waren, ein gefragtes Konsumgut, an das der Normalbürger zu DDR-Zeiten jedoch kaum herankam. Nach einer Phase des extremen Auftragsrückgangs erhielt der Betrieb 1990 durch einen alten Kunden Aufträge, die er aber auf Grund von Organisationsschwierigkeiten und einem Personalabbau von 110 auf 60 Personen kaum bewältigen konnte. Der Geschäftsführer und ehemalige Betriebsdirektor hatte die Anteile der ehemaligen Eignerin erworben und versuchte nun, die wertvolle Immobilie, auf der der Betrieb stand, in seine Hand zu bekommen und die Produktion so weit wie möglich zu reduzieren. Er zerstörte damit die wirtschaftliche Grundlage des Betriebs so vollständig, daß dieser im September 1992 von der Treuhand in die Liquidation geschickt wurde.

Die Forschung in dem dritten Betrieb, Hochhinauf, einer Aufzugsfirma, die 1990 von einer Westfirma übernommen wurde, begann im August 1991. Die strukturellen Veränderungen und der Personalabbau von 1000 auf 500 Beschäftigte wurde von der Käuferfirma vorgenommen, und auch für die Vermarktung wurden westliche Manager eingesetzt. In der Produktion blieb die Leitung bei verändertem Produktionsprofil zunächst weitgehend gleich. Die Auftragslage verbesserte sich 1991 zunehmend, aber die Fertigung konnte die verschärften Produktivitäts- und Preisvorgaben kaum bewältigen. Umfassende Neuinvestitionen in Produktionsmaschinen und in Fertigungs- und Verwaltungsgebäude wurden 1992 in Angriff genommen. Seit Januar 1993 wurde mit dem Transfer einer Produktionsabteilung von West- nach Ostberlin die Arbeitsorganisation umfassend umgestaltet. 
Regelmäßige Anwesenheî́ in den ersten beiden Betrieben seit Mai 1990 und in dem dritten seit August 1991 erlaubte es mir, die Erklärungen und Kommentare der Beschäftigten mit den ablaufenden Transformationen in Verbindung zu bringen. Über einen Zeitraum von nun drei Jahren begleitete ich Arbeiter und Angestellte bei ihrer Arbeit. Die ethnologische Methode der teilnehmenden Beobachtung erlaubte es, durch die Beobachtungen des Arbeitsablaufs, die ständige Anwesenheit auch in Momenten von Spannungen und Konflikten, die Teilnahme an Geburtstagsfeiern, Arbeitsbesprechungen und Belegschaftsversammlungen, die Erzählungen und Erläuterungen der Arbeitenden in Bezug zu ihrem Handeln zu setzen und die Selbstdarstellungen mit den Beurteilungen durch Andere zu konfrontieren. Um die Reaktionen auf die Transformationsprozesse zu verstehen, versuchte ich, die Ideologie und das Funktionieren der Planwirtschaft innerhalb des Betriebs zu rekonstruieren. Ich mußte dabei feststellen, daß die Beschäftigten die Vergangenheit schon in einer Weise interpretierten, die ihrem Selbstbild für die Zukunft entsprach. Der sozialistische Wettbewerb, an dem fast jede Brigade noch bis 1989 teilgenommen hatte, wurde als völliger Blödsinn und als ganz und gar unwichtig hingestellt. Schriftliche Quellen, wie Brigadetagebücher, Protokolle von Partei und Gewerkschaftsversammlungen wurden zu wertvollen Gedächtnisstützen, wenn ich nach den Aspekten des realsozialistischen Alltags fragte, die am leichtesten vergessen wurden. Der bester Weg, um die Veränderungen am Arbeitsplatz zu ergründen, war es, sich den gesamten Arbeitstags lang an einem bestimmten Arbeitsplatz aufzuhalten. Dort erklärten mir die Arbeitenden gewöhnlich ihre Verrichtungen, traten in Interaktion mit Vorgesetzten und Kollegen, forderten Material und Werkzeuge an, monierten und/oder behoben Fehler und Versäumnisse von direkten Kollegen und anderen Abteilungen, erzählten Anekdoten aus dem Betriebsleben zu DDR-Zeiten, erläuterten mir ihre politischen Einstellungen vor und nach der Wende und ihre politischen Einschätzungen zum Stand der deutschen Vereinigung, berichteten über aktuelle Probleme im Betrieb oder in der Familie, kommentierten die Auftragssituation und die Anforderungen des gerade von ihnen bearbeiteten Auftrags, analysierten die Betriebspolitik der Geschäftsführung usw.

\section{Der Marktwaln}

Der Produzent war König in der Planwirtschaft. Trotz verschiedener Versuche in den siebziger Jahren, sich den Wünschen der Konsumenten verstärkt anzupassen, biieb die Planwirtschaft bis zum Schluß über einen Produktionsplan definiert, der nach politischen Zielsetzungen erstellt wurde 
und sich nur an zweiter Stelle nach den Wünschen der Konsumenten richtete. Obwohl die Staatsbetriebe in der DDR auch über Kosten von Material, Arbeitskräften und von Gemeinkosten Buch führten, waren die Preise, die den Konsumenten in Rechnung gestellt wurden und die in der Planabrechnung zählten, nicht einfach von den Produktionskosten abgeleitet, noch waren sie Marktpreise. Sie waren in gewisser Weise politische Preise. Der Industrieabgabepreis, mit dem die Betriebe in der Planabrechnung kalkulierten, bestand aus dem Betriebspreis und einer Produktionsabgabe, einer Art Luxussteuer, die von einer externen Preiskommission festgelegt und direkt vom Staat bezogen wurde. Auch wenn ein Betrieb für das nichtsozialistische Ausland produzierte, konnte er das Vier- bis Fünffache des Inlandspreises des gleichen Produkts in seine Planabrechnung schreiben. Vor dem Fall der Mauer hatte Taghell zum Beispiel drei Arten von Kunden: die iniändischen Konsumenten, die Kunden aus dem nicht-sozialistischen Ausland und die Spezialaufträge von Regierungsinstitutionen und Parteimitgliedern. Die Lampentypen für den inländischen Gebrauch richteten sich nicht nach dem Geschmack oder der Nachfrage der ostdeutschen Konsumenten, sondern nach dem vorsichtigen Ausbalancieren der finanziellen Planverpflichtung und der Verpflichtung, die richtige Menge zu produzieren. Die finanziellen Verpflichtungen waren am leichtesten dadurch zu erfüllen, daß eine relativ kleine Menge von sehr teuren Gütern, belegt mit einer hohen Luxussteuer, produziert wurden, wie zum Beispiel Messingarmleuchter. Um die vorgeschriebene Menge zu erreichen, wurden diese dann durch große Serien von Lampen ergänzt, die man mit wenig Aufwand fertigen konnte, wie zum Beispiel einfache Blechlampen. Für eine schwedische Firma hatte der Betrieb elegante Messingleuchter entworfen und produziert, die auch in westdeutschen und westeuropäischen Kaufhäusern angeboten wurden. Für den Export der Lampen nach Schweden wurden dem Betrieb von der staatlichen Außenhandelsgesellschaft große Summen für die Planerfüllung gutgeschrieben.

Die Partei- und Regierungsinstitutionen bestellten außerhalb des Plans Lampen für Prestigeobjekte, wie Luxushotels, Ministerien und Gästehäuser der Partei. Die Beschäftigten schätzten diese Sonderaufträge sehr, denn sie erhielten dafür eine besondere Bezahlung und wurden ohne Probleme mit allem nötigen Material versorgt. Der für diese Spezialaufträge einst zuständige Abteilungsleiter erklärte mir, immer ein gern gesehener Gast in den von ihm belieferten Hotels gewesen zu sein.

Jeder Betrieb mußte selbst mit der Mangelwirtschaft zurechtkommen, innerhalb des Betriebes durch Horten und Sparen und nach außen durch Tausch mit anderen Betrieben und durch die Aktivierung persönlicher Netzwerke. In den Kanälen der grauen Ökonomie bestimmte sich der 
»Wert« des Produktes nach seiner Bedeutung als Investitionsgut oder nach seine Beliebtheit als Konsumgut. Dementsprechend waren die Tauschpartner bereit, sich Mühe zu geben.

In der Planwirtschaft gab es drei Formen der Verteilung: die offiziell geplante Verteilung, die nutzlos war für die Feinabstimmung; die politischbürokratische Verteilung, die auf persönlichen Beziehungen entlang der Parteilinien basierte; schließlich die pragmatische Redistribution, die den direkten Tausch von dringend benötigten Investitions- und Konsumgütern zwischen Firmen und Individuen umfaßte. Ein wirtschaftliches Verhalten, das dem der Marktlogik nahegekommen wäre, war nicht erforderlich, auch nicht in den beiden informellen Wegen, sich Güter zu beschaffen oder sie zu verteilen.

Der Fall der Mauer schaffte zunächst ein Vakuum in der Verantwortung für die Verteilung und Kommerzialisierung von Gütern. Die staatlichen Vermarktungsorganisationen hörten auf zu funktionieren oder vertrieben hauptsächlich Westprodukte. Die Manager der DDR-Betriebe waren plötzlich mit der Aufgabe konfrontiert, ihre Produktion auch zu vermarkten. In Betrieben wie Taghell, die einen soliden westlichen Geschäftspartner hatten, und in Bereichen wie bei Stanex, in denen ein begehrtes Investitionsgut produziert wurde und die in der DDR-Wirtschaft sehr erfolgreich gewesen waren, gab es vorsichtigen Optimismus. Die Manager erklärten, daß sie froh über das Verschwinden der schwerfälligen Staatsbürokratie wären und daß sie nun in der Lage sein würden, ihre wahren Fähigkeiten unter Beweis zu stellen.

»Und da haben wir mit unseren 10 Millionen 2,5 oder 3 Millionen an Gewinn reingebracht und da hat auch der Leiter einen großen Anteil mit dran, durch seine Arbeit in der Projektierung, Konstruktion, auch zu uns hin usw., daß wir immer versucht haben, dieses eine Produkt, diese Maschine immer mehr zu systematisieren. Und damit werd ich natürlich immer billiger, ist doch ganz klar! ... Damit, glaub ich, waren wir auch ein bißchen vorbildlich - in unserem Betrieb vor allen Dingen, und auch gar nicht schlecht im allgemeinen in der alten DDR Wirtschaft, indem wir dieses Prinzip durchbrachten und damit ökonomischer wurden. Und wir hätten uns - wenn die Bedingungen damals marktwirtschaftliche gewesen wären - wir hätten uns da eigentlich auch behaupten können.« (Produktionsvorbereitung, Stanex, 7.5.1991)

Der Leiter der Produktionsvorbereitung von Stanex hob die Effizienz und die Rationalisierungserfolge des Bereichs Montageautomaten zu DDR-Zeiten hervor und folgerte daraus, daß er erfolgreich in der Marktwirtschaft hätte wirtschaften können. Er beurteilte die ökonomische Leistungsfähigkeit jedoch mit Zahlen aus der Planabrechnung und maß sie somit an einer weitgehend fiktiven Größe, die für die Marktwirtschaft keinerlei Aussagekraft besaß. Nicht nur DDR-Betriebe sondern auch westliche Investoren überschätzten so die Chancen der Betriebe in der Marktwirtschaft. 
Bis April 1990 lebten die meisten DDR-Betriebe in der Illusion, daß sie für die nächsten beiden Jahre gefüllte Auftragsbücher hätten. Mit dem Näherrücken der Währungsreform vom 1. Juli 1990 begannen die Befürchtungen zu wachsen, daß ihr Produktionsniveau nicht mit dem von westlichen Firmen konkurrieren könnte. Um sich für die Marktwirtschaft vorzubereiten, begannen die Leiter vor allem die Schwächen ihrer Betriebe zu verbessern, die sie bisher vor den Kontrollinstanzen der Planwirtschaft verborgen hatten: die niedrige Produktivität und die variable Qualität des Produktes. Sie schätzten ihre Chancen in der Marktwirtschaft auf der Grundlage der Probleme ein, die sie in der Planwirtschaft gehabt hatten, und fuhren fort, den Schwerpunkt ihrer Geschäftspolitik auf die Restrukturierung der Produktion zu legen.

Zwei von den drei Geschäftsführungen zogen es vor, die Produktion ohne die Intervention eines westlichen Geschäftspartners zu reformieren. Als der schwedische Großkunde von Taghell im Frühjahr 1990 eine Fusion vorschlug, weigerte sich der Geschäftsführer, in diese Art der engen Kooperation einzutreten, die seine Macht und seine Kontrolle über den Betrieb untergraben könnte. Der Geschäftsführer von Stanex reagierte ähnlich, als 1990 mögliche Käufer begannen, den Betrieb zu besuchen. Obwohl eine Fusion oder ein Kauf die wirtschaftliche Situation des Betriebes möglicherweise verbessert hätte, so hätte er doch den Einfluß der Manager erheblich eingeschränkt, die 1990 noch in einem Machtvakuum operieren konnten, das kleinen und mittleren Betrieben von der Treuhand gelassen worden war. Durch die Ausnutzung von Treuhand-Krediten und durch die Möglichkeit Kurzarbeit anzumelden, standen die beiden Betriebe bis Ende 1990 unter relativ geringem finanziellen Druck. Die Finanzen des untersuchten Bereichs von Stanex wurden von der Buchhaltung der Mutterfirma mitverwaltet, die im Laufe des Jahres 1991 langsam bankrott ging und deren Schulden im Sommer 1992 beim Verkauf an eine Immobilienfirma von der Treuhand übernommen wurden. Taghell lebte von Krediten der Treuhandanstalt, die der Betrieb nicht zurückzahlen konnte. Nur die dritte Firma Hochhinauf trat schon ab November 1989 in enge Kooperation mit drei westlichen Partnern, von denen einer die Firma später übernahm.

Als viele Konsumenten in Ostdeutschland nach dem 1. Juli 1990 plötzlich die DDR-Produkte ablehnten, die sie in der Vergangenheit so hoch geschätzt hatten, traf das viele Produzenten wie ein Schock. Die Geschäftsführer von Lampengeschäften, die früher Taghell angefleht hatten, ihnen Lampen zu verkaufen, weigerten sich nun, noch etwas von ihnen zu beziehen. $\gg$ Die Leute in der Vertriebsabteilung werden so für die Arroganz bestraft, die sie früher an den Tag gelegt haben «, meinte ein Mitarbeiter dazu. Hochhinauf, das von einer amerikanischen Firma vor allem wegen seiner 
Serviceverträge übernommen worden war, verlor im Sommer $199040 \%$ der Wartungsverträge an konkurrierende Firmen, weil sich die Kundien an den schlechten Service erinnerten, der ihnen geboten worden war, als die Firma noch das Monopol für Personenaufzüge in der DDR besaß. Es bleibt unklar, wie die alten Kunden von Stanex reagiert hätten, wenn sie neue Rundschalttischautomaten gebraucht hätten. Zu Zeiten der Planwirtschaft hatte Stanex ihnen jeglichen Service für Reparaturen und Ersatzteile verweigert und ihnen stattdessen die technischen Zeichnungen der Automaten gegeben, die sie dann selbst reparieren mußten. Wie dem auch sei, die ostdeutsche elektrische und elektronische Industrie, ihr Hauptkunde, hatte seit Mai 1990 fast alle Bestellungen storniert und war seit dem Sommer 1990 in einer so kritischen Lage, das sie nicht an Investitionen denken konnte.

Nicht nur die Reaktion der ostdeutschen Kunden, sondern auch die Einführung von Produkten auf dem westdeutschen Markt bereitete dem Management und den Angestellten von Taghell und Stanex Probleme, die nicht nur rein wirtschaftlicher Natur waren. Die Entwicklung einer Marketingstrategie und das Herstellen erster Kontakte mit möglichen westlichen Kunden, die von den Angestellten der Vertriebsabteilung vorangetrieben wurden, wurden vom Management immer wieder an zweite Stelle gesetzt. Noch im Sommer 1990, nach der Währungsreform, verbat der Direktor des von mir untersuchten Bereiches von Stanex den Angestellten der Vertriebsabteilung, die Namen von potentiellen Kunden bei der Westberliner Industrieund Handelskammer nachzuschlagen. Er hing noch an der alten politischen Aufteilung der Welt und wollte sein Produkt nur Kunden in Ostdeutschland und in Osteuropa zugute kommen lassen.

$\gg D a$ hat sich der Doktor kraft seiner Wassersuppe durchgesetzt und hat uns... naja indirekt vergewaltigt, daß wir eben die ehemaligen DDR-Betrjebe aufsuchen. Aber zu dem damaligen Zeitpunkt... uns allen war im Prinzip klar, da kann nichts rauskommen, daß das (der ökonomische Zusammenbruch B.M.) jetzt praktisch die Runde machen würde.... da sind wir teilweise richtig am verschlossenen Betriebstor gewesen, wo 'ne Kette rumgelegt war und es nicht mal mehr einen Pförtner gab. (Projektierungsleiter Stanex 2.5.1991)

Als sich seine Hoffnungen auf den osteuropäischen Wirtschaftsraum völlig zerschlagen hatten und er keine Kunden bekam, stimmte der Direktor im Herbst $1990 \mathrm{zu}$, Briefe an potentielle Kunden im Westen zu schicken und ihnen sein Produkt vorzustellen. Die Briefe waren jedoch immer noch im alten Befehlston geschrieben, den sich die Produzenten in den alten Tagen der DDR gegenüber den Konsumenten erlauben konnten. Dennoch erhielt er einige interessierte Anfragen, aber nur eine von ihnen führte schließlich zur Bestellung eines Rundschalttischautomaten. Erst im Frühling 1991 mobilisierte er alle Ingenieure, die er entbehren konnte, um mögliche Kunden in Westdeutschland aufzusuchen. Sie wurden nach Angeboten gefragt, aber kein definitiver Auftrag folgte. Im Sommer 1991, als schon $80 \%$ der 
Belegschaft auf Kurzarbeit war und der Direktor der Besitzer des Unternehmens geworden war, akzeptierte er schließlich, auch kleinere und andersartige Aufträge anzunehmen. Die Beziehungen zwischen ostdeutschen Produzenten und westdeutschen Konsumenten hatten sich inzwischen radikal gewandelt, seit der erste Enthusiasmus über die deutsche Vereinigung verflogen war.

»Am Anfang war eigentlich ein relativ gutes Gefuhh, war der ganze Trend positiv... als wir dort Interessenten aufgesucht haben, die waren höflich und korrekt gewesen... so '90. Waren alle eher angetan. Man hat gemerkt, da war noch ein bißchen diese Euphorie dagewesen. Man hat uns Mut gemacht: 'Na klar, das schaffen wir schon gemeinsam.' Aber schon im Dezember rum fing der Trend an, so langsam umzuschlagen. Daß man vielleicht doch schon erkannt hat, daß man, daß wir in gewissem Sinne eine Konkurrenz darstellen können, daß wir vielleicht. doch was können und nachher nicht bloß als, naja weiß ich nicht,... Almosenempfänger dastehen an der Stelle. Und jetzt ist der Trend eigentlich, naja teilweise schon so, daß man fast das Gefühl hat, es wird einem ein bißchen feindlich begegnet... Es ist sogar schon aufgetreten, als wir zu Partnern hingekommen sind, daß die gesagt haben, wir sollen sie nicht stören, sie müssen ja für uns mitarbeiten. Wir wollen ja doch bloß höhere Löhne und Gehälter haben, arbeiten wollen wir nicht und so. ' Macht mal ein bißchen schnell. Ihr stört uns bloß bei der Arbeit'«. (Projektierungsleiter Stanex 2.5.1991)

Der Verkäufer nahm die Weigerung, bei ihm etwas zu kaufen, als eine persönliche Ablehnung durch seine westdeutschen Geschäftspartner wahr. Die ost-westdeutschen Vorurteile beeinflußten die Interpretation der Verkaufssituation. Der ostdeutsche Verkäufer hatte das Gefühl, daß er sich gegen das Bild eines Almosenempfängers zur Wehr setzen mußte oder, wie ein anderer Verkäufer meinte, gegen das eines Bettlers. Er setzte aber gleich-

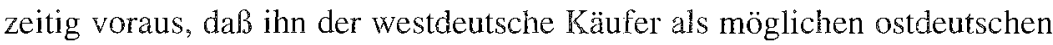
Konkurrenten fürchtete und deshalb ablehnte. Indem er auf die Verkaufssituation den Verdacht projizierte, daß Westdeutsche absichtlich Ostdeutsche in der Stellung von zweitklassigen Bürgern halten wollen, fühlte er sich wie ein unerwünschter Konkurrent, obwohl sein Betrieb in keiner Weise mit dem des potentiellen Kunden konkurrierte.

Die Aussagen verschiedener Verkäufer stimmten darin überein, daß die ostdeutschen Hersteller während der ersten Monate nach dem Fall der Mauer mit dem psychologischen Vorteil der Neugierde und des emotionalen Engagements ihrer westdeutschen Geschäftspartner rechnen konnten. Dieser Effekt verschwand, als die Kosten und Probleme der Vereinigung immer klarer wurden und als die Blüte der ostdeutschen Wirtschaft immer mehr in die ferne Zukunft rückte.

Offenbar existierte ein Widerspruch zwischen der Wahrnehmung und der Realität des Marktes. Am Anfang schienen die Beschäftigten der von mir untersuchten Betriebe der Auffassung gewesen zu sein, daß der Markt wie ein hartes, aber doch faires System funktioniere, welches die Fähigen und Hartarbeitenden belohnt und die Faulen und Nachlässigen bestraft. Die jet- 
zige Desillusionierung rührt wohl daher, daß der ehrliche Produzent nicht unbedingt am Markt erfolgreich ist, daß er vielmehr häufig von einem Konkurrenten mit einer gefälligen Marketing-Strategie, auch wenn diese kaum durch die Qualität des Produkts gerechtfertigt ist, ausgestochen wird.

\section{Der Mythos yom faulen Ossi}

Ein Standardelement der westlichen Vorurteile sozialistischen Wirtschaftssystemen gegenüber war, daß ein Mangel an Arbeitsdisziplin in den Betrieben die natürliche Faulheit der Arbeiter beförderte und daß Faulheit nun bei ostdeutschen Arbeitern Gewohnheit geworden sei. Als ich jedoch 1991 und 1992 Arbeiter und Angestellte in ostdeutschen Betrieben interviewte, unterstrichen diese gerade die Qualitäten, die sie nach westdeutschem Vorurteil nicht haben sollten: Sorgfalt, Disziplin und Eigeninitiative. Die Frauen in der Montageabteilung von Taghell zum Beispiel bestanden darauf, daß sie die von ihnen montierten Leuchter mit größter Sorgfalt prüften. Die Meister bei Stanex hoben ihren Witz und ihre Autorität über die Arbeiter hervor, während die Arbeiter in der selben Abteilung ihre Fähigkeit betonten, unabhängig vom Meister zu produzieren und sich auf ihre eigene Schläue und Fertigkeiten zu verlassen. Die Arbeiter von Hochhinauf betonten 1991, daß sie ihr Bestes gaben und verdächtigten das westliche Management, sie bis zur äußersten Grenze ihrer Leistungsfähigkeit treiben zu wollen. Wie sind diese Unterschiede in der Wahrnehmung zu erklären?

Die Arbeitsorganisation in der Planwirtschaft ähnelte der von E.P. Thompson für vorindustrielle Gesellschaften beschriebenen aufgabenorientierten Produktion. Nichtstun wechselte sich ab mit Phasen intensiver Aktivität (Thompson 1967, 73). Lange Perioden mit Maschinenstillstand wegen Materialmangel oder Mangel an Ersatzteilen wechselten sich ab mit Phasen von Nachtarbeit und Wochenendschichten. In Zeiten weniger intensiver Aktivität zogen sich die Arbeiter der Vorfertigung bei Taghell gerne in ihre verschiedenen Frühstücksecken zurück oder, wenn sie nicht gestört werden wollten, zu ihrer Ecke unten am Fluß, die durch ein Tor verborgen war, zu dem einer der Kollegen den Schlüssel hatte. Dennoch, wenn gegen Ende des Monats alle Abteilungen begannen, besonders hart zu arbeiten, um den Monatsplan zu erfüllen, dann zogen auch sie mit. Ihr Engagement ging weit über die Verpflichtung, Überstunden zu machen, hinaus. Sie arbeiteten Wochenenden durch und in Doppelschichten, fuhren die Maschinen bis an ihre äußerste Grenze und ließen dabei die Sicherheitsbestimmungen außer acht. Der Meister erzählte mir unter dem Siegel der Verschwiegenheit, daß sie manchmal in den Nachtschichten die rumänische 63t-Presse, die nach unten unkontrolliert ausschlug, zum Nieten von Leuchterarmen 
benutzt hatten. Dies sei zwar sehr gefährlich, aber auch sehr effizient gewesen. In stiller Übereinkunft mit dem Meister beteiligten sich die Arbeiter an der besonderen Anstrengung, gegen Monatsende den Plan zu erfüllen, aber sie erwarteten dafür, am Anfang des nächsten Monats in Ruhe gelassen zu werden. Voskamp und Wittke $(1991,31)$ nannten diesen Interessenkompromiß treffend den $\gg$ Planerfüllungspakt .

Der Gebrauch von alter, schwer zu kontrollierender Technologie trug de facto zur Autonomie der Arbeiter bei. In den drei Betrieben waren die meisten Maschinen zwanzig bis dreißig Jahre alt. Fin hohes Maß an Improvisation war nötig, um die Maschinen am Laufen zu halten und den Mangel an Ersatzteilen und adäquatem Material auszugleichen. Die Arbeiter von Stanex, die spezielle Komponenten für den Rundschalttischautomaten ferígen mußten, erreichten einen Grad an Genauigkeit, der es mit dem von modernen Maschinen aufnehmen konnte. Diese Präzision zu erreichen war nur möglich, weil die Arbeiter ihre Maschine und ihre Defekte seit langem kannten und sich darauf einstellen konnten. Dieses Qualitätsniveau zu erreichen war zeitaufwendig, und die Arbeiter konnten daher ihren Arbeitstag recht frei gestalten. Sie hatten zwar eng kalkulierte formelle Arbeitsquoten, die aber durch großzügige Einrichtzeiten ausgeglichen wurden.

Die Arbeitsorganisation in der Planwirtschaft förderte zwei typische, sich nicht ausschließende Reaktionen bei den Beschäftigten. Sie regte die Arbeiter und Angestellten an, die Grenzen ihrer Freiheit auszutesten und so wenig wie möglich zu arbeiten, aber sie ermutigte sie auch, auf ihre eigene Initiative hin zu arbeiten, an der Arbeit selbst Gefallen zu finden und stolz auf ihr Produkt zu sein.

»...Hähnchen im Härteofen gegrillt, Eisbein gekocht und Sahne auf dem Bohrwerk geschlagen. Aber es hat niemandem geschadet. Die haben alle hinterher wieder gearbeitet. Das mag ja nun für einen westlichen Menschen ein bißchen komisch anmuten, aber genau das war es ja, was eigentlich die Sache hier lebenswert gemacht hat auf seine Weise. Wir haben ja ewig Iange in diesem Betrieb gehockt, von sieben bis halb fünf früher. Und so war es erträglich. Es war ja wirklich ne dufte Truppe... Aber die Nischen, die hier gesucht wurden und die eigentlich das angenehme Leben gemacht haben, die waren auch auf Arbeit. Die waren nicht nur in der Freizeit. Und das waren teilweise schöne Nischen, muß ich sagen. (Facharbeiter, Stanex, 15.6.1991)

Die Arbeiter von Stanex, die sich an diesen Streichen und Provokationen beteiligten, gingen in anderen Momenten völlig in ihrer Arbeit auf. Wenn sie zum Beispiel im letzten Montageschritt den zwölf Stationen des Automaten $»$ Leben einhauchten«, arbeiteten die zwei verantwortichen Arbeiter, der mechanische und der Elektrikkonstrukteur, oft Tag und Nacht. Die Zusammenarbeit zwischen Facharbeitern und Ingenieuren, die schon bei der täglichen Arbeit intensiv und unabhängig von formalen Hierarchien war, wurde fast eine Symbiose in diesen privilegierten Momenten. In den letz- 
ten fünfzehn Jahren konnten sie dank dieser spontanen Kooperation einen Industrieautomaten entwickeln, der es mit westlicher Technologie durchaus aufnehmen konnte.

Das System der Planwirtschaft entmutigte eine fortlaufende Verausgabung von Arbeit und ermutigte das Wachstum einer unproduktiven Bürokratie, aber es stimulierte gleichzeitig ein erstaunliches Maß an Eigeninitiative und Improvisation bei den Beschäftigten. Arbeit in Verwaltung und Produktion, die als unangenehm und überflüssig angesehen wurde, wurde nur widerwillig erledigt, während viel Mühe in die Beschaffung von seltenen Materialien oder in die Entwicklung von neuen Produkten investiert wurde. So hatten Angestellte von Hochhinalf gemeinsam mit Beschäftigten anderer Betriebe eine regelmäßige informelle Materialtauschbörse organisiert. Die Planwirtschaft funktionierte in erheblichem Maße dank des persönlichen Engagements und der besonderen Anstrengungen der Beschäftigten, die im Plan nicht vorgesehen waren.

Threr Selbsteinschätzung nach hatten die Beschäftigten nicht nur versucht, den Plan zu erfüllen, sondern sie waren es, die das Funktionieren des Systems gewährleisteten, indem sie mehr als verlangt gegeben hatten. Das verbreitete westliche Vorurteil, »die Ossis sind faul und unproduktiv«, lief also der Selbstwahrnehmung der ostdeutschen Beschäftigten diametral entgegen, da sie sich auch im Vergleich zu den Arbeitenden der anderen Länder des östlichen Blocks als die leistungsfähigsten und diszipliniertesten begriffen hatten.

»Eh, dieser Slogan so ungefähr 'na die blöden Ossis, die können nichts, die sind nichts, das zieht sich ja über alle Bereiche. Das ist ja nicht so als wärs-, sagen wir mal industriebezogen, sondern-, ob das Lehrer sind, ob das Ärzte sind, ob das Krankenschwestern sind-; die taugen solange nichts, bis nicht Bedarf bei irgendwelchen westlichen Firmen oder Einrichtungen besteht. Die da arbeiten können. Dann sind sie auf einmal eigenartigerweise gut ausgebildete Leute. Wenn man sie brauchen kann. Aber viele Unternehmer, wie gesagt, die wissen das auch, $d a ß$ die Leute, daß die, daß die sich noch zu helfen wissen... weil wir uns die ganze Zeit immer was einfallen lassen mußten...eben aus der Mangelwirtschaft heraus... Diese Schutzbehauptung (die Ossis sind faul B.M.) - ich seh sie mehr als eine Schutzbehauptung von alteingesessenen Bundesbürgern an - , die auch logischerweise Angst vor der Konkurrenz haben. Denn wir stellen eine Konkurrenz da. Eindeutig! Wir drücken, wir drücken von hier aus auch ganz schön mit (auf die Löhne B.M.)《 (Facharbeiter 18.4.1991)

Dieser Facharbeiter dreht die Bezichtigung der Faulheit um und wertet sie als Ausdruck der Eifersucht und der Angst der westdeutschen Beschäftigten vor ihren potentiellen ostdeutschen Konkurrenten auf dem Arbeitsmarkt. Nach seiner Interpretation müssen sich nicht die Ostdeutschen vor den Westdeutschen rechtfertigen, sondern die Westdeutschen müssen sich vor der Leistungsfähigkeit der Ostdeutschen in acht nehmen.

Die hohe individuelle Produktivität, die ostdeutsche Arbeiter in der Lage sind zu erreichen, wenn sie in westdeutschen Unternehmen arbeiten, steht 
in scheinbarem Widerspruch zu der niedrigen Produktivität der meisten ostdeutschen Betriebe. Die Erklärungen vieler ostdeutscher Manager für dieses Phänomen ähneln dem westlichen Vorurteil. Sie beklagen sich über den Mangel an Disziplin im Betrieb und versuchen eine striktere Kontrolle über ihre »faule« Arbeiterschaft auszuüben. Arbeiter und Angestellte aller drei Betriebe auf der anderen Seite betonen ihre hohe individuelle Produktivität und die Unfähigkeit der Manager, das Unternehmen zu führen.

Vom Herbst 1990 an entließen der Direktor von Stanex und der Geschäftsführer von Taghell einen großen Teil ihrer Beschäftigten. Auswahlkriterium für die verbleibende Belegschaft waren Freundschaftsbeziehungen, Parteizugehörigkeit und alte Seilschaften im Betrieb. Qualifikationen und Berufserfahrungen schienen bei der Auswahl zweitrangig gewesen zu sein. In der Montage von Taghell beklagten die Frauen im Herbst 1990 die verschärften Arbeitsnormen und die willkürlichen Entlassungen. Zu Zeiten der Planwirtschaft war es auch im Interesse des Meisters und des Gesamtbetriebes gewesen, daß die Frauen eine Norm von $115 \%$ nicht überschritten, weil andernfalls die Planvorgaben für das kommende Jahr zu stark erhöht worden wären. Aus Furcht vor Entlassungen erhöhten die Frauen nun von sich aus ihre individuelle Arbeitsleistung bis zu einer Normerfüllung von 140 bis $145 \%$. Als die Entlassungen trotzdem weitergingen und die Normen noch mehr erhöht wurden, kehrten die Frauen im Frühjahr 199l zur kollektiven Kontrolle ihrer Arbeitsleistung zurück, diesmal gegen das Interesse und den Willen ihrer Vorgesetzten.

Bei Stanex wurde ab Winter 1990 größerer Wert auf die Einhaltung tayloristischer Tugenden, wie Pünktlichkeit, Ordnung und Respekt der Vorgesetzten, gelegt, aber die Arbeitsorganisation blieb weitgehend gleich. Da die formale Hierarchie in der Fertigung nicht mit einer Hierarchie der Erfahrung und der Qualifikation übereinstimmte, fuhren die Arbeiter fort, nicht ihre Meister bei Produktionsproblemen zu konsultieren, sondern sich direkt mit den Ingenieuren abzusprechen. Die Arbeiter und Konstrukteure schafften es dennoch im Frühling 1991, einen Automaten innerhalb von drei Monaten zu konstruieren und zu fertigen, für den sie vor dem Fall der Mauer ein Jahr benötigt hätten.

Bei Hochhinauf verbesserte sich die Marktlage zusehens im Frühling 1991, während die Produktionsprobleme zunahmen. Die Großserien von DDRAufzügen wurden gestoppt und Einzelaufträge für Spezialaufzüge wurden hereingenommen. Dieser Übergang von der Massenproduktion zur Produktion von Einzelstïcken und Kleinserien, die eine flexible Anpassung an den Markt erlaubte, brachte Probleme mit sich, die Hochhinauf mit fast der gesamten ostdeutschen Industrie teilte (Voskamp und Wittke 1992a). Statt 
Teil eines hoch differenzierten Produktionsprozesses zu sein, sollten die Arbeiter nun ganze Aufzugskabinen in kleinen Arbeitsgruppen montieren. Als ich den Mechanikern im Herbst 1991 beim Montieren der Kabinen zusah, verbrachten sie einen großen Teil ihres Arbeitstages mit der Suche nach Material, dem Sortieren von technischen Zeichnungen, mit der Diskussion ihres Vorgehens und dem Nachmessen der zugeschnittenen Teile. Oft kam das Material, das sie als erstes brauchten, als letztes, weil die Koordination zwischen Montage und Zuschnitt schwerfällig war. Die Arbeiter entdeckten häufig einen Fehler in den technischen Zeichnungen erst, als die Montage schon weit fortgeschritten war. Konstruktionsfehler, wie zum Beispiel ein Sitz, der unter dem Handlauf plaziert worden war, so daß sich niemand mehr darauf setzen konnte, veranlaßte die Arbeiter zu Heiterkeitsausbrüchen. Mit der Formel, »die werden dafür bezahlen«, verwiesen sie auf die Verantwortung der Konstrukteure.

Die Arbeiter beschwerten sich, daß sie, obwohl sie nun Einzelstücke und keine Serien mehr produzieren sollten und wesentlich mehr unterschiedliche Arbeitsschritte zu tun hatten, weniger Unterstützung von der Konstruktion und der Arbeitsvorbereitung bekamen als früher. Auf den Konstruktionszeichnungen, nach denen zum Beispiel die Schweißer vor der Restrukturierung der Produktion Plattformen gebaut hatten, waren die Schweißfolgen und auch einige Vorrichtungen angegeben, die für die einzelnen Arbeitsschritte zu benutzen waren. Diese waren nun nicht mehr auf den Zeichnungen des Westberliner Konstruktionsbüros angegeben.

Die Fertigung erreichte die im Preis kalkulierten Fertigungsvorgaben nicht. Die Arbeiter hatten dadurch zwar keine finanziellen Nachteile, weil sie Stundenlohn erhielten, aber sie hatten das Gefühl, daß die von ihnen verlangten Leistungen völlig unrealistisch gewesen seien. Sie hatten aber gleichzeitig Angst, daß sie, falls die von ihnen nicht erreichten Produktivitätsziele doch eine reelle Grundlage hätten, um ihren Arbeitsplatz fürchten müßten. Auf der anderen Seite hatten die westlichen Manager, die mit der Aufgabe betraut waren, das Unternehmen zu restrukturieren, den Verdacht, daß die Beschäftigten absichtlich ihre Leistung zurückhielten, weil sie laut Tarifvertrag Ost nur 60\% des Westlohns erhielten.

Das Produktivitätsproblem erwies sich als ein organisatorisches, denn es hörte schlagartig auf, als zum 1. Januar 1993 die Fertigung aus Westberlin mit der in Ostberlin zusammengelegt wurde. Die Organisation der Fertigung wurde völlig neu strukturiert, die Aufgaben der einzelnen Arbeiter waren nun klar umrissen, die Zuarbeit aus anderen Abteilung funktionierte besser. Innerhalb von drei Monaten war der Produktionsriickstand des letzten Jahres aufgeholt und die ostdeutschen Arbeiter erreichten die gleiche Produktivität wie ihre westlichen Kollegen. Die ostdeutschen Beschäf- 
tigten hatten nun die Gewißheit, daß sie es mit ihren westdeutschen Kollegen aufnehmen konnten und daß ihr Arbeitsplatz nicht direkt in Gefahr war. Aber sie erhielten nur die Hälfte der Einkünfte, die den Arbeitern aus dem Tarifgebiet West bis Ende 1994 garantiert worden waren, mit denen sie nun Seite an Seite arbeiteten.

Obwohl eine unterschiedliche Bezahlung nun nicht mehr mit Produktivitätsargumenten zu rechtfertigen war, schloß sich das Management von Hochhinauf Anfang April 1993 dem Bruch des Tarifvertrages Ost an und zahlte den ostdeutschen Arbeitern zu den vom Arbeitgeberverband angebotenen neun Prozent Erhöhung nur eine sechsprozentige betriebsinterne Zulage. Beim ersten Warnstreik der IG Metall, dem drei Viertel der produzierenden Beschäftigen aus dem Osten folgten, schlossen sich die Kollegen aus dem Westen nicht an, obwohl sie nun auch zum Tarifgebiet II gehörten. Die neue Lohnpolitik ist darauf angelegt, Gräben zwischen ostdeutschen und westdeutschen Arbeitern aufzuwerfen, die in der ersten Phase der Zusammenarbeit erfolgreich vermieden worden waren. Die ostdeutschen Beschäftigten bleiben trotz aller guten Worte von Seiten des Managements Mitarbeiter zweiter Klasse, die sich bisher noch in diese Position fügen, weil sie kaum andere Chancen auf dem Arbeitsmarkt hätten. Der ostdeutsche Arbeiter ist auch bei Hochhinauf kein König mehr.

\section{Schlußfolgerung}

Ich versuchte darzustellen, mit welchen Ideen die Ostdeutschen der Marktwirtschaft beigetreten und welches die Gründe für ihre heutige Frustration und Enttäuschung sind. In den von mir untersuchten Betrieben konnte ich vor allem zwei Faktoren ausmachen: Der erste war die Erwartung, daß die Marktwirtschaft wie ein »ehrliches« Systen funktionieren würde, das die Fähigen belohnt und die Nachlässigen bestraft. Nach zwei Jahren Marktwirtschaft mußten die Beschäftigten von Taghell und Stanex feststellen, daß ihre Strategie, sich dem Markt anzupassen, indem sie die Qualität ihrer Produkte verbesserten und die Produktivität erhöhten, vergeblich war. Sie bekamen das Gefühl, als Produzenten betrogen worden zu sein. Die Enttäuschung schien vor allen in der Tatsache gelegen zu haben, daß sie feststellen mußten, daß die besten Produzenten nicht unbedingt diejenigen waren, die ihr Produkt am besten vermarkteten.

Nach vierzig Jahren Planwirtschaft, in denen sie gezwungen waren, die Schimäre des Plans zu verteidigen, wollten viele Ostdeutsche nun eine moralische Erneuerung ihrer Gesellschaft. Sie erwarteten sie in der Marktwirtschaft, von der sie angenommen hatten, daß sie ohne falsche Vorspiegelungen und nach objektiven Gesetzen funktionieren würde. Als dieses Bild 
des Marktes zerbrach, wurde auch ihre Rolle als Konsumenten ambivalent. Die Ostdeutschen mußten feststellen, daß die Freuden des Konsums längst nicht so groß waren, wie sie gehofft und erwartet hatten. Sie erfuhren, was Hirschman $(1982,46)$ dargestellt hat: immer wenn der wirtschaftliche Fortschritt für bestimmte Schichten den Zugang zu Konsumgütern erweitert hat, kommen starke Gefühle der Enttäuschung oder der Feindseeligkeit gegenüber dem neuen materiellen Reichtum zum Vorschein. Nachdem die Ostdeutschen eine Revolution gemacht haben, um an den Genüssen der Konsumgesellschaft teilzuhaben, beschleicht sie nun der Verdacht, daß sie es vielleicht nicht wert war.

Der zweite Faktor war, daß die von mir interviewten Arbeiter und Angestellten beklagten, daß selbst wenn sie es geschafft hatten, ihren Arbeitsplatz zu behalten, sie doch die Gewißheit verloren hatten, daß ihre Rolle in der Gesellschaft eine wichtige war. Arbeiter und Angestellte fühlten, daß sie an ihrem Arbeitsplatz austauschbar geworden waren. Sie empfanden die plötzliche Auflösung der komplexen sozialen und politischen Rollen, die ihnen die offizielle DDR-Ideologie zugeschrieben hatte, nicht nur als Erleichterung, sondern auch als Verlust. Die Arbeitsbeziehungen, die sich ungeplant in der Planwirtschaft entwickelt hatten, wurden nun mit Nostalgie erinnert. Arbeiter und Angestellte mußten feststellen, daß ein gewisser Freiraum, der sich in den Nischen der Planwirtschaft entwickeln konnte, verschwunden war. Die Zeit, die sie nun im Betrieb verbrachten, war zwar kürzer, aber sie war mehr auf Leistung ausgerichtet und weniger mit sozialem Leben erfüllt.

Die Unsicherheit der Beschäftigungssituation, ein kritsches Bewußtsein für die politische Vergangenheit und die heutigen Fehler und Versäumnisse ihrer alten/neuen Vorgesetzten, hohe Ansprüche an die Flexibilität der Arbeiter und ein Mangel an Transparenz in der Restrukturierung der Produktion führten zu Demotivation und manchmal zu passivem kollektiven Widerstand der Beschäftigten. Die Angst vor dem Verlust des Arbeitsplatzes oder dem endgültigen wirtschaftlichen Niedergang des Betriebs ließen bisher die Proteste der ostdeutschen Beschäftigten eher leise ausfallen. Nur kurz vor dem endgültigen Aus ihres Betriebs erhoben sie meist noch einmal die Stimme. Der jüngste Streik der IG Metall könnte die Situation verändern und den Bundesbürgern klarmachen, daß die ostdeutschen Beschäftigten keine Lust mehr haben, zweitklassige Bürger zu sein. Die Annäherung der beiden Tarifgebiete ist mehr als eine Frage des Geldes. 


\section{Literatur:}

Belwe, K. 1979: Mitwirkung im Industriebetrieb der DDR, Opladen

Belwe K., Klinger F. 1986: Der Wert der Arbeit: Aspekte des sozialen Wandels in der Arbeitswelt der DDR, in Tradition und Fortschritt in der DDR, Deutschland Archiv 19

Bust-Bartels, A.1980: Herrschaft und Widerstand in DDR Betrieben, Frankfurt a. M.

Etzioni, A. 1988: The Moral Dimension. Toward a New Economics, New York: Free Press

Godelier M. 1984: L'idéel et le matériel: Pensée, economies, societés. Paris: Fayard

Gorz, A. 1983: Wege ins Paradies. Thesen zur Krise, Automation und Zukunft der Arbeit, Berlin

Gorz, A. 1989: Kritik der ökonomischen Vernunft, Berlin

Hann, C.(ed.) 1990: Market Economy and Civil Society in Hungary, London

Heine H.J. u.a. 1990: Die Zukunft der DDR Wirtschaft, Reinbek

Hirschman, A.O. 1989: Entwicklung, Markt und Moral München

Hirschman, A.O. 1982 Shifting Involvements, Cambridge

Hirschman, A.O. 1991: Management und Belegschaft im wirtschaftichen Wandel - Brüche und Kontinuitäten, unveröffentlicher Beitrag zu der Tagung »Kontinuität und Wandel in ostdeutschen Betrieben «, Universität Bielefeld 25. und 26. Oktober 1991

Lüdtke, A. 1991: 'Ehre der Arbeit': 1ndustriearbeiter und Macht der Symbole. Zur Reichweite symbolischer Orientierungen im Nationalsozialismus, in Tenfeld K. (Hg.) Arbeiter im 20. Jahrhundert, Stgt.

Marz, L. 1991: Der prämoderne Übergangsmanager. Die Ohnmacht des 'realsozialistischen' Wirtschaftskaders, in Deppe R., Dubiel H., Rödel U. (Hg.) Demokratischer Umbruch in Osteuropa Frankfurt/M.

Müller, B.1991: Toward an Alternative Culture of Work. Political Idealism and Economic Practices in West Berlin Collective Enterprises. Boulder, Col.: Westview Press

Müller, B. 1991: Einbruch der Marktwirtschaft in die Alltäglichkeit von zwei Betrieben in der ehemaligen DDR, in Sozialanthropologische Arbeitspapiere, Nr. 37

Müller, B. 1992: De la 'folie du marché' à la fin des illusions, in Liber. Supplément aux actes de la recherche en sciences sociales, 91-92, p.14-15

Müller, B. 1992: De la compétition socialiste à la libre concurrence: la privatisation de trois entreprises du peuple (VEB) à Berlin Est, in Allemagne aujourd'hui, Nr. 121

Müller, B. 1993: The Wall in the Heads: East-West German Stereotypes and the Problems of Transition in Three Enterprises in East Berlin, in Anthropological Journal on European Culture, Vol. 2, Nr.1

Niethammer, L., von Plato A., Wierling, D. 1991: Die volkseigene Erfahrung. Eine Archäologie des Lebens in der Industrieprovinz der DDR, Berlin

Popitz H., Bahrdt H.P., Kesting E.A. 1972: Das Gesellschaftsbild des Arbeiters, Tübingen

Rottenburg, R. 1991: Der Sozialismus braucht den ganzen Menschen. Zum Verhältnis vertraglicher und nichtvertraglicher Beziehungen in einem VEB, in Zeitschrift für Soziologie, Jg. 20 , Heft 4

Sabel, C.F. 1982: Work and Politics, Cambridge

Scott J. 1990: Domination and the Arts of Resistance. Hidden Transcripts, New Haven

Thompson E. P. 1967: Time, Work Discipline and 1ndustrial Capitalism, in Past and Present, No. 38

Voskamp U., Wittke V. 1991: Aus Modernisierungsblockaden werden Abwärtsspiralen - zur Reorganisation von Betrieben und Kombinaten in der ehemaligen DDR, in Berliner Journal für Soziologie 1

Voskamp U., Wittke V. 1992: Junge Facharbeiter in der Produktion - eine Herausforderung für betriebliche Arbeitspolitik, in SoFi Mitteilungen 19

Voskamp U., Wittke V. 1993: Den Westen überholen ohne ihn einzuholen? Zu den Schwierigkeiten bei der Restrukturierung der ostdeutschen Industrie und den Perspektiven erfolgversprechender Reorganisationsstrategien, in R. Schmidt (ed.) Zwischenbilanz: Analysen zur industriellen Transformation, Berlin 